\title{
MedienPädagogik
}

www. medienpaed.com

Zeitschrift für

Theorie und Praxis

der Medienbildung

ISSN 1424-3636

Themenheft Nr. 23: Visuelle Kompetenz

\section{bildbild - visuelle Kompetenz im Unterricht}

Norbert Grube, Thomas Hermann, Sarah M. Schlachetzki und Kurt Caviezel

\begin{abstract}
Der vorliegende Artikel stellt das Webseiten-Projekt bildbild vor. ${ }^{1}$ Der Webseite liegt die Motivation zugrunde, Schülerinnen und Schüler für ein Nachdenken über globale Themen zu sensibilisieren. Ein solches Nachdenken ist immer auch eines über bildlich vermittelte Wirklichkeit. Es ist mit bildbild ein Anliegen, angesichts einer allseits konstatierten «Bilderflut» ein didaktisches Medium zu entwickeln, das einen Fundus an historischen und aktuellen Fotografien zur Verfügung stellt und diese so aufbereitet, dass sie gerade nicht hinter ihrer illustrativen Funktion verschwinden. Dadurch sollen die Bilder für ein selbständiges Lernen auf dem Weg hin zu einer visuellen Kompetenz einsetzbar gemacht werden. Der Beitrag beleuchtet die Entwicklung der Webseite vor dem Hintergrund kulturkritischer Haltungen zum Bild seit der Moderne sowie im Rahmen dessen, was seit den 1970er Jahren im pädagogischen Kontext als Visual Literacy diskutiert und gefördert wird. Dabei wird auch auf die medialen Potenziale einer Webseite für die Bildlesekompetenz von Jugendlichen eingegangen.
\end{abstract}

Jedes Nachdenken über Bilder beginnt mit dem Betrachten. Die mussevolle Betrachtung ist jedoch nicht selbstverständlich angesichts des vielfältigen, fast unendlich grossen Angebots an Bildern. "Wir alle sind trainiert im schnellen Anschauen von Bildern, weil wir anders mit der Bilderflut um uns herum nicht fertig werden können. Wenn wir dagegen ein Bild vor unseren Augen sozusagen anhalten und es über die vorab zugebilligte Zeit betrachten, kommt das zustande, was wir den gedehnten Blick nennen können. Der gedehnte Blick sieht auch dann noch, wenn es nach allgemeiner Übereinkunft, die schon längst beim nächsten und übernächsten Bild angekommen ist, nichts mehr zu sehen gibt» (Genazino 2004, S. 42). Wie also den "gedehnten Blick» erlernen? Wie gerade Schülerinnen und Schülern die Möglichkeit geben, das Bild als Bild zu erfahren, ohne bei der portionierten Bildbetrachtung etwa im Kunstunterricht zu verharren oder über die

1 http://bildbild.ch/. Die Entwicklung einer Webseite benötigt zeitliche und finanzielle Ressourcen. Wir sind dankbar, dass die Abteilung Forschung und Entwicklung der PH Zürich uns die Arbeit an dieser Webseite ermöglicht hat. Ebenso zur Realisation beigetragen haben Drittmittel von Seiten der Stiftung Bildung und Entwicklung (http://www.globaleducation.ch/). 
blosse Forderung nach reflektierterem Einsatz von Bildmaterial im Unterricht nicht hinaus zu kommen?

\section{Bildkritik von der Geburtsstunde der Fotografie zum Pictorial Turn}

Forderungen nach einer Kultur des Sehens sind nicht neu. Sie koinzidieren gehen mit der Entwicklung moderner Bild- und Reproduktionstechniken einher und fallen damit in die Hochphase kulturpessimistischer Debatten kurz nach der Jahrhundertwende 1900. Die einen feiern dabei ab dieser Zeit die Potentiale der Fotografie und kehren mit der Betonung einer internationalen sozialdokumentarischen Fotografie (Stumberger 2010, S. 10 f., 14) ihre politische Bedeutung hervor, so auch Kurt Tucholsky in seinem Aufruf nach «Mehr Fotografien!» von 1912. Diesseits und jenseits politischer Grabenkämpfe hält die Fotografie seit ihrer Frühzeit soziale Umstände fest und wird zum ethnographischen Medium. So erhält sie wenig später als ethnographische Methode zusammen mit dem Film systematisch Einzug in die Sozialwissenschaften - als Blick auf «das Fremde` wie auch auf alltagskulturelle Phänomene (Christolova 2011, S. 149-150). Mit der Vermarktung der handlichen Leica ab 1925 trägt der Fotojournalismus erheblich zu jener neuen Vergegenwärtigung von Welt bei, die ebenso gefeiert wie kritisiert wird. Denn neben den Anhängern der Fotografie warnen wiederum andere vor ihr als Teil der aufgekommenen Massengesellschaft, als Medium der Massennivellierung und trügerischer Suggestivkraft. Nicht zuletzt sind es die («technischen») Bilder, die für die moderne Reizüberflutung, Überforderung und Nervosität der Gesellschaft verantwortlich gemacht oder aber - vor dem Hintergrund der Radikalisierungen des frühen zwanzigsten Jahrhunderts - für politische Zwecke missbraucht werden können (Radkau 1998, S. 277; Nolte 2000, S. 309). ${ }^{2}$

Es wird die Entfremdung des Menschen von seiner Umwelt bedauert, die durch den Relevanzverlust eigener Erfahrungen entsteht, durch die Dominanz der nun aufkommenden «Erfahrungen zweiter Hand` für das Verstehen von Welt. So leisten Bilder, etwa von Pin-up Girls, laut Arnold Gehlens Diktum der Erfahrungsverarmung einer Emotionalisierung zweiter Hand Vorschub (1957/2004, S. 44-54, 66f.; 1952/2004, S.226). Nüchtern scharfsinnig, aber in gewisser Parallele zu Gehlen konstatiert später Susan Sontag (2011, S. 148, 154), dass Fotografie eine Besitzergreifung von Realität und damit zugleich Ausdruck eines Kompensationswunsches nach Vereinfachung der Welt und neuen Erfahrungen sei: «Das Fotografieren ist eine Methode zum Einfangen einer als widerspenstig und unzulänglich empfun-

2 Zentral hierzu Benjamins Kunstwerk Aufsatz, 1936/2003. Benjamin betont, dass anders als beim Kunstwerk eine notwendige "freischwebende Kontemplation» beim Betrachten von Fotos (als Bilder nun gleichsam «Beweisstücke») nicht mehr adäquat sei, S. 21. Siegfried Kracauer hingegen nimmt bereits 1927 in seinem Aufsatz «Die Photographie» eine ambivalente Position bezüglich ihrer Wirkung ein, indem er sie einerseits als «Generalinventar der [...] Natur» (Kracauer 1990, S. 96) beschreibt, andererseits ihren Einsatz in den Illustrierten als «mächtigstes Streikmittel gegen die Erkenntnis» moniert (ebd., S. 93). 
denen Realität. Oder sie ist ein Mittel zur Vergrösserung einer Realität, die geschrumpft, ausgehöhlt, vergänglich, weit weg wirkt.» (Sontag 2011, S. 156).

Seit dem beginnenden 20. Jahrhundert entwickelt sich eine nicht nur auf Europa beschränkte, sondern auch in den USA laut vernehmbare Kritik an zunehmend visuellen Massenmedien (u. a. durch John Dewey oder Walter Lippmann; siehe Grube 2012, S. 200): sie beförderten, so heisst es, Verdummung, seichte Zerstreuung, Oberflächlichkeit, Passivität und mangelnde Information. Diese Sichtweise greifen etwa seit den 1960er Jahren Theodor Adorno und Jürgen Habermas wieder auf, indem sie gerade das Fernsehen, aber auch die ihrer Aufklärungsfunktion entsagenden, affirmative «Show» bietenden Massenmedien kritisieren (Adorno 1970; Habermas 1990, S. 307, 310, 319). Massenmedial - und damit auch immer bildlich - konsumierte Unterhaltung lenke den an sich aufgeklärten Bürger von Engagement und Partizipation in der Politik ab. «Hyperrealität〉 und «Simulacrum» werden im französischen Sprachraum zu Inbegriffen einer Realität der Bilder, die realer als die Wirklichkeit selbst geworden sei (Baudrillard 1981, S. 12-17; 123-124).

Demgegenüber hat sich, seit geraumer Zeit unter dem Schlagwort des Pictorial Turn, gleichsam eine Bildeuphorie Bahn gebrochen: Bilder sorgten für Transparenz, Teilhabe, Sichtbarkeit von Welt und hätten - mit Blick etwa auf aufrüttelnde Fotos aus dem Vietnamkrieg (Arnold 2010, S. 323) - politisch aufklärende, mobilisierende Relevanz (ganz in Einklang mit frühen Positionen wie etwa jener Tucholskys Jahrzehnte zuvor). Inmitten der für demokratisch-kapitalistisch verfasste Gesellschaften notwendig erachteten Zirkulation von Waren und Wissen könnten Bilder eine herausragende Rolle einnehmen. Sie ermöglichten Zugänge zur Welt (Boehm 2007, S. 14). Fotografien dehnten das «Reich des Sichtbaren beträchtlich aus», wie selbst Sontag $(2011$, S. 111, 149) zugesteht. Sie zeigten verdichtete Prozesse der Globalisierung.

Die Kritiker der Fotomassenware und die Anhänger des Pictorial Turn haben eines gemeinsam: sie forderten und fordern eine Bildung und Kultur des Sehens: die einen, um hochkulturelle Kunstwerke und kontemplative Vertiefung zu erhalten; die anderen, weil sie «Bildverstehen als Kulturverstehen in medialisierten Gesellschaften» (Soeffneru. Raab 2004, S. 249) erachten und um «Bilderlesen als gleichwertige Kulturtechnik wie das Lesen von verbalen Texten zu positionieren» (Doelker 2011, S. 10).

\section{Historische Verortung des Begriffs Visual Literacy}

Vor dem Hintergrund der Debatten um die Bildermaschinerie als oberflächliches Amüsement, Diskussionen um Bilderlügen und -manipulation, Nachrichtenflut und visuelle Überforderung wird in den USA seit Jahrzehnten das Konzept einer Visual Literacy gefördert. Der Begriff hat Konjunktur und ist in aktuellen Publikationen zur Integration von Bildern in schulische Kontexte durchaus präsent. So fordert Ulrich 
Schnakenberg (2012) in seiner Monografie zur «Karikatur im Geschichtsunterricht» zu allererst eine "Ausbildung von «visual literacys als Reaktion auf die Bilderflut».

Bereits 1969 fasste John Debes den Begriff sehr weit als eine «Reihe von Sehfähigkeiten, (. . .) die eine visuell kompetente Person dazu befähigt, sichtbare Handlungen, Objekte, natürliche oder von Menschen geschaffene Symbole (...) wahrzunehmen, zu unterscheiden und zu interpretieren.» (Debes 1969, S. 27) ${ }^{3}$

Zu den Zielen einer Visual Literacy zählt die Definition Debes' eine geförderte Kommunikationsfähigkeit sowie das Vermögen, wie es wörtlich heisst, «to comprehend and enjoy the masterworks of visual communication» (Debes 1969, S. 27) und - damit einhergehend - kritisches Reflektieren. Sie schliesst ausdrücklich die sichtbare, primäre Wirklichkeit mit ein, das heisst ebenso zwei- wie auch dreidimensionale Objekte. Eine diesbezügliche Einschränkung des Begriffs auf visuelle Medien, wie sie etwa Christian Doelker (2011, S. 12) vorschlägt, ist für schulische Zwecke sinnvoll. Im Übrigen ist Debes' Definition so offen formuliert, dass sie durchaus anschlussfähig für weiterführende Auseinandersetzungen mit einer Visual Literacy ist. Spätere Definitionen aus dem angelsächsischen Raum heben den einen oder anderen Aspekt etwas mehr hervor oder erweitern das Spektrum um einzelne Fähigkeiten. So wird etwa postuliert, dass visuelle Kompetenz die Fähigkeit beinhalte «Objekte vor dem geistigen Auge herauf[zu]beschwören» (Brill et al. 2000; zitiert in Sims et al. 2002, S. 2). Das Oakland Museum of California, das mit «Picture This» didaktische Materialien zur Förderung von Visual Literacy online bereitstellt, definiert den Begriff mit Bezug auf die Schule:

«Visual literacy is defined as the ability to understand communications composed of visual images as well as being able to use visual imagery to communicate to others. Students become visually literate by the practice of visual encoding (expressing their thoughts and ideas in visual form) and visual decoding (translating and understanding the meaning of visual imagery).» http://museumca.org/picturethis/visual.html (2003)

Im deutschen Sprachraum wird der Begriff «Visuelle Kompetenz» als Übersetzung des englischen verwendet. Der Medienpädagoge Christian Doelker hat mit seinem Buch «Ein Bild ist mehr als ein Bild - Visuelle Kompetenz in der Multimediagesellschaft» (1997) einen umfassenden Beitrag zur Bildsemantik vorgelegt. Ausgehend von seinem Modell der drei Wirklichkeiten (primäre, mediale und subjektiv wahrgenommene Wirklichkeit) und über die Zuschreibung verschiedener funktionaler Bedeutungen arbeitet er ein differenziertes Schichtenmodell von Bildbedeutungen heraus. Für eine schulische Förderung von visueller Kompetenz schlägt Doelker ein «Bilderlesen in drei Schritten» vor (Doelker 2011, S. 24). In einem ersten Schritt schreibt der betrachtende Schüler, die Schülerin dem Bild

3 Aus Debes' Engagement und dem einiger Kollegen ging in dieser Zeit ebenfalls die Gründung der International Visual Literacy Association (http://ivla.org/) hervor. 
subjektive Bedeutung zu. Im zweiten Schritt spricht gleichsam das Bild: hier geht es um Offenlegung der dem Bild inhärenten Bedeutung. In einem dritten Schritt schliesslich kommen mit der Frage nach der intendierten Bedeutung die Bildautorin, der Bildautor bzw. bildinterpretierende Experten mit Kontextinformationen zu Wort (Doelker 2011, S. 25).

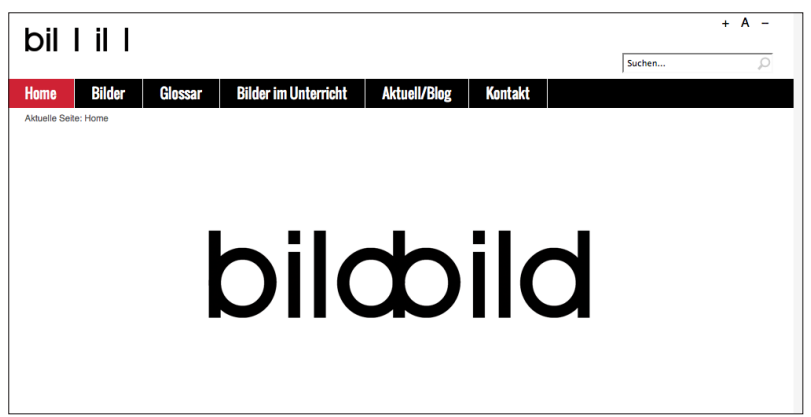

Abb. 1: Logo auf der Einstiegsseite von www.bildbild.ch

\section{3 bildbild: Entwicklung einer Webseite}

Bei der Entwicklung der Webseite bildbild (Abb. 1) wurden im Rahmen eines umfassenden Verständnisses von Visual Literacy zwei Einschränkungen vorgenommen: Erstens beschränkt sich die Webseite auf «man-made symbols» - wobei der Bezug zur realen Lebenswelt zentral ist. Zweitens fokussiert bildbild auf das Medium Fotografie. Die Beschränkung auf Fotografie hat mehrere Gründe. Sie ist als Medium in allen Bereichen von Gesellschaft, Wissenschaft und Kunst präsent. Entsprechend gross ist die Vielfalt innerhalb ihrer Anwendungsbereiche: Von privaten Handy-Schnappschüssen im Alltag von Jugendlichen, über fotografische Dokumentationen und Spurensicherungen, bildgebende Verfahren in Medizin und Naturwissenschaften, massenmedial verbreitete Newsbilder aus allen Sparten des öffentlichen Lebens, anonyme, automatisch aufgenommene und wieder verschwindende WebCam-Bilder bis hin zur Kunst ist Fotografie als visuelles Ausdrucks- und Erkundungsmittel unendlich wandelbar. Diese Adaptions- und Anschlussfähigkeit in funktionale Bezüge moderner Gesellschaften, Wissenschaften und Wirtschaft erklärt sicherlich auch die globale Verbreitung der Fotografie. Die einzige Schwäche von Fotos ist möglicherweise die, dass sie - mit Gottfried Boehm gesprochen - als die «unverbrüchlichen Garanten ... [einer] nützlichen Verdoppelung der Welt gesehen» (Boehm 2007, S. 43) - und damit gleichzeitig oft übersehen - werden. Fotografien aber auf ihre Abbildfunktion zu reduzieren heisst, sie nicht als gestaltete Bilder zu würdigen und sie mit ihrem kommunikativen Potential sowie in ihrer Konstruktion von Wirklichkeit nicht ernst zu nehmen. 
Mit Blick auf das Schulfeld, mit Blick also auf den Unterricht und auf die Mediensozialisation und Identitätsentwicklung von Kindern und Jugendlichen, ist die Fotografie ein zentrales Medium. Lehrmittel der meisten Fächer sind reichlich mit Fotos illustriert. Auch wenn im Unterricht unterschiedlich darauf Bezug genommen wird, so sind Fotos doch mehr nur als «motivierende Goodies»: Sie prägen in Fremdsprachlehrmitteln die Vorstellungen anderer Länder und Kulturen mit; schaffen einen Bezug zwischen der Lebenswelt der Lernenden und derjenigen ihrer Altersgenossen in anderen Sprachregionen. Geschichtslehrmittel sind mit Fotos zur neueren Geschichte gespickt. Dass diese nicht immer als Quellen thematisiert und schon gar nicht als visuelle Verdichtungen dekonstruiert werden, bringt Heuer auf den Punkt, wenn er mit Genazino für den "gedehnten Blick» in der Geschichtsdidaktik plädiert (Heuer 2006). Bilder als Stabilisatoren und Rückkoppelungen von Diskursen verweisen auf ihre Funktion als Abbild (von Vergangenem) und Denkbild (aktueller Konstruktionen) zugleich. In Lehrmitteln für die Naturwissenschaften tragen Fotos zu Verständnis und Erkenntnisgewinn bei (Merzyn 1994, S. 137, 236) und vermitteln gleichzeitig ein Bild der Naturwissenschaften, sind also «Images of Science» im doppelten Sinne des Wortes. Werbe- und Pressebilder werden in der Medienbildung auf kommunikative Strategien oder nach Kriterien wie Gender oder Bildmanipulation hin diskutiert. Ebenso wichtig für das Schulfeld schliesslich sind Fotos als Medien der Erinnerung (z. B. Klassenfotos, vgl. Grosvenor, Lawn u. Rousmaniere 1999) und als Mittler in Aushandlungsprozessen von Identitäten - seien es Fotos von Sport-, Musik- oder Filmidolen, seien es Selbstporträts im Bildertausch zwischen Jugendlichen.

Trotz eines regen Interesses an Fotografie und dem Bewusstsein um deren mannigfaltige Potentiale ist das Angebot an Materialien zur Fotografie in der Schule, das über die Bebilderung von Lehrmitteln hinausginge, nicht sehr gross. Dieser Bedarf zog die Idee für bildbild nach sich, um ein Instrument für eine differenzierte Auseinandersetzung mit Fotografie zu entwickeln, das sowohl für Schülerinnen und Schüler als auch für Lehrpersonen einfach zugänglich und nutzbar ist. Mit der Förderung von bilddidaktischen Kompetenzen von Lehrpersonen und der Bildkompetenz bei Schülern/-innen soll zugleich dem «Sozialisations-, Erziehungs- und Bildungswert bildhaften Materials in unterschiedlichen Kontexten pädagogischen Handelns» (Schäffer 2005, S. 213) neue Relevanz verschafft werden. Dreh- und Angelpunkt ist dabei das Bild für Lern-, Reflexions- und Verständigungsprozesse, die über seine reine Illustrationsfunktion hinausreichen. Es soll darum gehen, inhaltliche und formale Bildelemente und -strukturen kennen zu lernen, zu erkunden, zu vergleichen, möglicherweise neu arrangieren zu können und in Bezug zu sprachlichen Ausdrucksmöglichkeiten zu setzen. Denn die «visuelle Verknüpfungslogik von Bildaufteilung und Farbgebung, von Linienführungen und Lichtverhältnissen sowie der Anordnung von Objekten und Personen begründet den optischen Mehrwert 
bildlicher Darstellungen und eröffnet dem Betrachter Erkenntnischancen jenseits des von ihm bereits Gewussten und deshalb Wiedererkannten. » (Soeffneru. Raab 2004, S. 267) Die Webseite bildbild möchte damit einen anderen als den «gedehnten Blick» auf das Bild erschweren und im intensiven Betrachten und Vergleichen eine reflektierte Teilhabe an der Welt als einer Welt der Bilder ermöglichen. Zu diesem Zweck stellte die Fotostiftung Schweiz ihre einmalige Sammlung zur Verfügung, aus deren Bildfundus bestimmte Bildserien ausgewählt wurden.

Erste explorative Befragungen von sechs Lehrpersonen verschiedener Schultypen im Vorfeld der Webseitenentwicklung ergaben zwei Bedarfslagen für die Arbeit mit Bildern im Unterricht: Einigen Lehrpersonen geht es primär um die Stärkung der visuellen Kompetenz von Schülerinnen und Schülern, d. h. um eine Sensibilisierung für die Wirkungsweisen von Bildern mehr oder weniger unabhängig von konkreten Lerninhalten. Andere Lehrpersonen äussern vor allem fachlich motiviertes Interesse an inhaltlicher Arbeit mit einer Webseite zu fotografischen Bildern. Dieser doppelte Anspruch - erstens die Förderung von visueller Kompetenz, zweitens eine inhaltliche Auseinandersetzung mit Themen des Globalen Lernens - war somit Ausgangspunkt für den Weg von der Auswahl des Einzelbildes bis hin zur grafischen Umsetzung der Benutzeroberfläche von bildbild (Abb. 2).

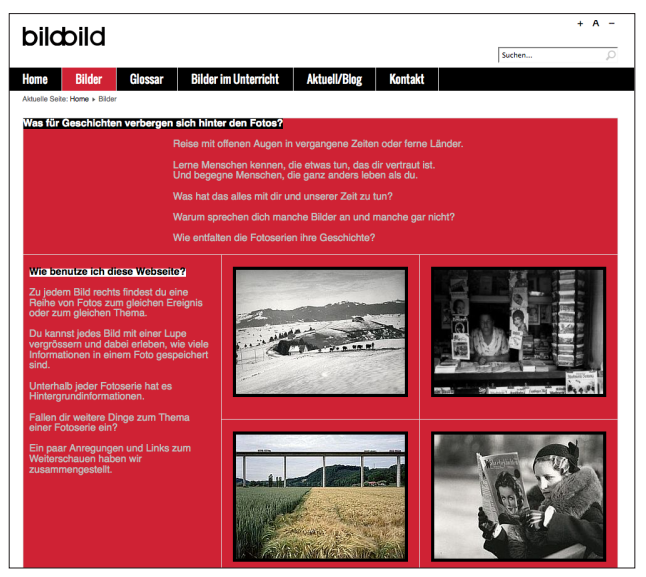

Abb. 2: Einstiegsbilder aus dem Menu-Punkt «Bilder» laden zum Entdecken ein.

Diesen Gedanken versuchen die in einem ersten Schritt für bildbild zusammengestellten Bildstrecken Rechnung zu tragen, indem folgende global, fotodokumentarisch und zugleich lebensweltlich aktuelle relevante Themen berücksichtigt wurden:

- Migration: Flucht, Arbeitssuche, Umsiedelung

- Natur und Umwelt: Menschliche Eingriffe in Landschaften

- Medien: Mittler zwischen Menschen und Welten 
Ziel der Auswahl ist es, sowohl die Einzelbildbetrachtung und -deutung zu vertiefen, als auch die vergleichende Betrachtung und Analyse von Bildserien zu ermöglichen (Abb. 3). Dieser Zweischritt ist in der Erziehungs- und Bildwissenschaft mit der seriell-ikonografischen Fotoanalyse (Pilarczyku. Mietzner 2005) als Methode konzeptionalisiert und etabliert worden und kann - durch bildbild entsprechend didaktisch aufbereitet - im Unterricht angewendet werden.

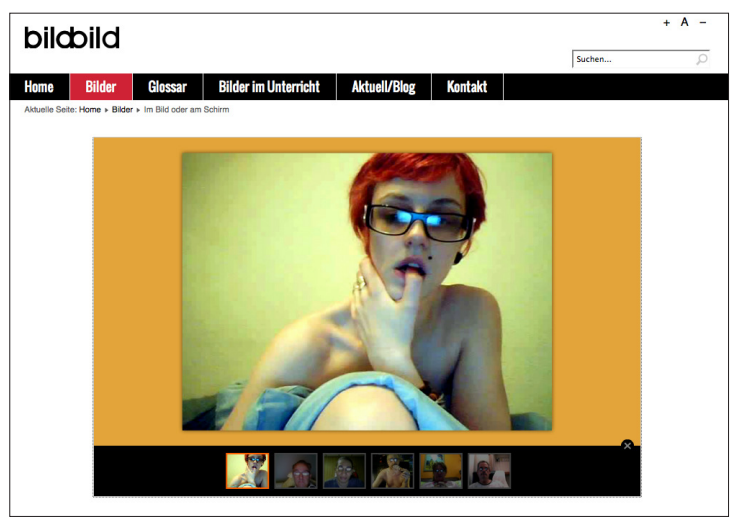

Abb. 3: Das Einzelbild (gross) im Kontext einer Serie (hier im Bild unten, klein)

Das Anliegen, medienspezifische Vorteile einer Webseite für die Arbeit mit dem fotografischen Bild nutzbar zu machen, findet in der Konzeption der digitalen Oberfläche Niederschlag: Eine Zoomfunktion erlaubt das instantane Vergrössern von Bilddetails - ein Aspekt, der bei der Arbeit mit gedrucktem Bildmaterial unmöglich ist. Per Mausklick in die Bilder hinein und hinaus zu zoomen befähigt Schülerinnen und Schüler dazu, das Einzelbild als Quelle von Informationen zu erfahren, die sich mitunter erst auf den zweiten, verweilenden Blick erschliessen. Vom Einzelbild einer Fotoserie ergeben sich Bezüge zur gesamten Bildstrecke eines Themas. Einzelbild und Bildstrecke bilden somit die Einheit, von der die Unterrichtsarbeit mit der Webseite ausgeht. Eine Präsentationsfunktion in Vollbildmodus ergänzt die Zoomfunktion. Während das Einzelbild zunächst in seiner singulären Komplexität gewürdigt werden kann, eröffnet eine jede Fotoserie vielzählige inhaltliche und formale Anknüpfungspunkte. Der Blick soll für genau diese Bezüge geschärft werden, indem die Schülerin, der Schüler in eigenständigem Erkunden die Funktionalität der Webseite zu nutzen lernt. Didaktische Fragen leiten die Lernenden an, die Einzelbilder in ihrem formalen Aufbau, ihren perspektivischen Zugriffen auf globale Themen und ihren narrativen Strategien wahrzunehmen. ${ }^{4}$ Einzelbild um

4 Für eine erste Vertiefung in die einzelnen Bilder schlagen wir die Methode der «Visual Thinking Strategy" (VTS) vor. In Zusammenarbeit von Museen und Schulen wird VTS in den USA seit einigen Jahren als Unterrichtsmethode eingesetzt, die neben der visuellen Kompetenz auch das kritische Denken sowie das Zuhören und die Ausdrucksfähigkeit von Schülerinnen und Schülern fördert. 
Einzelbild setzt sich im klickenden, zoomenden Erforschen die jeweilige Fotoserie aus durchschnittlich sechs Fotos zusammen. Eingeblendete «Blickaufträge`verweisen auf Zusammenhänge zwischen Einzelbild und Serie. Jenseits dieser Steuerung der Bildwahrnehmung verzichtet bildbild auf Text unmittelbar am Bild. Dieses soll zuerst für sich stehen können und durch keine sprachlichen Filter - seien es auch nur Titel, Autor oder Entstehungsdatum - verstellt werden (Abb. 4-6).

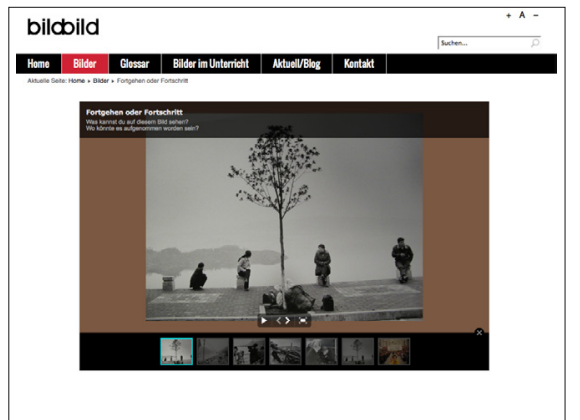

Abb. 4: Einzelbild mit eingeblendetem «Blickauftrag»

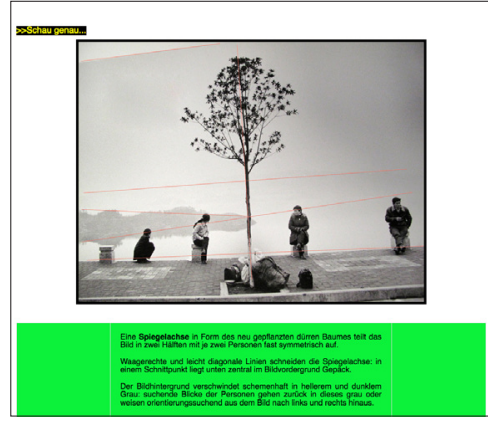

Abb. 6: Informationen und weiterführende Aufgaben zu den einzelnen Bildstrecken

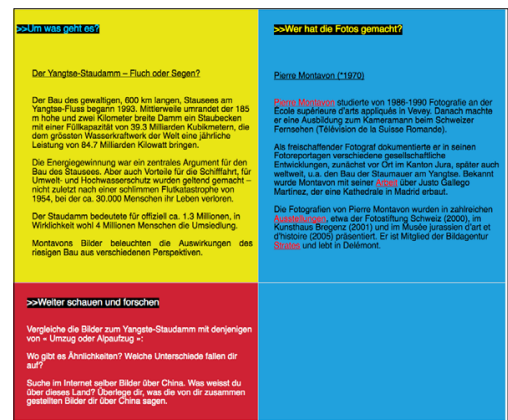

Abb. 5: Einzelbild mit Zusatzinformationen zu formalen, bildästhetischen Kriterien

Zusätzliche Informationen finden sich erst im Scrollen auf den unteren Teil einer Bildseite. Jeweils ein ausgewähltes Bild wird dort unter der Kategorie $>>$ Schau genau in seinen formalen Wirkungsweisen eingehender vorgestellt, damit verbunden werden fototheoretische Begriffe eingeführt, die auf einer übergeordneten Glossar-Seite Erklärung finden. Den Lernenden wird somit nach dem Erkunden einer Bildserie, das von eigenständigen Versprachlichungen des Gesehenen begleitet ist, ein Beispiel für sprachlich gerichtetes Schauen gegeben. Kontextinformationen zu den Bildern, Angaben zu Fotograf/in, Entstehungsdatum und -umständen der oftmals fotojournalistischen Arbeiten folgen weiter unten auf der Seite. An die Lebenswelt der Schülerinnen und Schüler knüpft die Kategorie $>>$ Weiter schauen und forschen an. Rechercheaufträge und Vorschläge zu selbstinitiierter Bildarbeit schlagen eine Brücke zwischen dem auf bildbild bereit gestellten Fotomaterial und aktuellen Themen für den Unterricht. Die durch die Fotoserien vorgestellten globalen Themen sollen dadurch an Alltagserfahrung und Lebenswelt der Kinder und Jugendlichen anschliessbar werden. Damit begegnet bildbild bildkritischen Positionen wie jenen Arnold Gehlens oder Jean Baudrillards, die eine defizitäre Information lediglich zweiter Hand oder die hyperreale Verstellung der eigentlichen Wirklichkeit beschreiben. Der Webseite bildbild hingegen liegt die Annahme zugrunde, dass jede Bildbetrachtung, Bildbeschreibung und Bilddeutung von zirkulierendem Wissen (Sarasin 2011) gelenkt wird, das seinerseits erheblich von Alltagserfahrungen geprägt ist. Die Fotos auf bildbild werden somit erstens als 
eigenständige Bildaussagen wahrgenommen, zweitens in Bezug zu anderen Fotos gesetzt und drittens mit ausserbildlichen Kontexten in Beziehung gebracht.

\section{Globales Lernen mit bildbild}

Um Jugendlichen die Teilhabe am «Diskurs ihrer Gegenwart, der in erster Linie ein Diskurs der Bilder ist» (Heuer 2006, S. 205), zu ermöglichen, werden auf bildbild Fotoserien aus unterschiedlichen Zusammenhängen aufbereitet, die untereinander vielfach anschlussfähig sind. Das globale Thema von Migration und Umsiedelung beispielsweise spiegelt sich in zwei verschiedenen Bildserien: Dem Lebensraum der Schülerinnen und Schüler räumlich nahe zeugt die Arbeit des Schweizer Fotografen Hans Staub (1894-1990) (Staub 1984) aus den 1930er Jahren vom Bau des Sihlstausees im Hochtal von Einsiedeln. Diese historischen Aufnahmen von Umsiedelung und Eingriffen in die Natur korrespondieren ihrerseits mit der aktuellen Fotoserie Pierre Montavons (*1970), die den Bau des Yangtse-Staudamms in China beleuchtet. Die Serien können im Unterricht einzeln zum Thema «Migration», ‘Umsiedelung〉, ‘menschliche Eingriffe in die Umwelt` bearbeitet werden - sie können aber auch aufeinander bezogen werden, um zum Verständnis historischer und globaler Konstanten beizutragen. Grossbauprojekte zum Beispiel, die eine massenhafte Umsiedelung von Menschen nach sich ziehen und die die Natur nachhaltig zum Wohle eines erhofften Fortschritts verändern, sind dabei ein Thema für den Geschichtsunterricht ebenso wie für Geographie oder Sozialkunde.

Durch zurückhaltende didaktische Akzentsetzungen sollen sich Schülerinnen und Schüler selbst ein Bild machen: inhaltlich, welche Geschichte in der Fotoserie wohl erzählt wird, sowie formal, d. h. mit welchen fotografischen Mitteln gearbeitet wird. Dadurch, dass die Kontextinformation zu den Serien erst weiter unten auf der jeweiligen Seite erscheint, sollen die Lernenden sich den Themen zunächst rein schauend und von ihrer eigenen Erfahrung ausgehend nähern können. Zur Erhärtung ihrer jeweiligen Vermutungen werden sie ermuntert, die gerade fokussierte Bildserie mit anderen Serien zu vergleichen, um dabei globale Gemeinsamkeiten zu entdecken. Neben Umsiedelung und Eingriffen in Natur und Landschaft greifen weitere Serien Themen wie Entwurzelung, Ortlosigkeit oder Mobilität auf. Seien es chinesische Wanderarbeiter in Andreas Seiberts ( ${ }^{\star 1970)}$ dokumentarischer Arbeit «From somewhere to nowhere» (Seibert 2008), seien es italienische Auswanderer nach Lateinamerika, die Yvan Dalain (1927-2007) in den 1950er Jahren fotografisch begleitete (Pfrunderu. Gasser 2003) - die Arbeit mit bildbild bietet eine Vielzahl von Anschlussmöglichkeiten an Inhalte des Fachunterrichts.

Seitdem der ungarische Maler, Fotograf und Lehrer László Moholy-Nagy die Schulung des Sehens durch Fotografie als «new instrument of vision» propagierte (1936/1981, S. 344), haben sich ihre technischen Möglichkeiten multipliziert. Die Fotografie als Ausschnitt der Wirklichkeit auf dem Negativfilm wird abgelöst von 
der medialen Zirkulation des Bildes, von Gebrauchsweisen des Fotos im Fernsehen und später im digitalen Raum des Internets. Die Auswahl des fotografischen Materials auf bildbild möchte Schülerinnen und Schüler auch für diesen Medienwandel sensibilisieren. Die Fotografin Yvonne Griss hat in den 1980er Jahren unter dem Titel «Melancholie» Menschen vor dem Fernseher ins Bild gesetzt. Sechs dieser wundersam entrückten Portraits regen zur Erörterung von Fernsehkonsum als Zeit der Stille, des aktiven Medienhandelns oder aber als passive Berieselung an. Damit ist zugleich ein klassischer Bildtopos aufgegriffen, der Menschen beim Schauen zuschaut. Eine Auswahl an Bildern aus dem riesigen digitalen Archiv des Fotokünstlers Kurt Caviezel wiederum macht heutige Gebrauchsweisen des fotografischen Bildes zum Thema, indem sie Menschen im anonymen Raum des Internets vor ihren WebCams zeigt (Abb. 1). Diese zeigende oder enthüllende Dimension des Bildes, das in den intimen Raum des Zuhauses vorrückt, wirft zugleich Fragen nach Voyeurismus und Internetnutzung auf. Sie stehen zwar häufig im Zentrum alarmierender Studien (Machill u.von Peter 2001), doch ihre Omnipräsenz können Schülerinnen und Schüler, angeregt durch Bildstrecken auf bildbild, einmal aus anderer, eigener Perspektive kritisch überdenken. Es geht um das «private), digitale Bild als tatsächlich oder oft nur vermeintlich ephemeres Bild, das in den Weiten des Internets ein erstaunlich langes Nachleben finden kann. Wie die Befunde der JAMES-Studie 2012 zeigen, ist das Hochladen von Fotos und Videos die am häufigsten angewandte Praxis im Bereich des «aktiven Gestaltens von Content im Internet» (Willemse et al. 2013, S. 31). Zugleich empfinden es aufgrund weit verbreiteter Erfahrungen Jugendliche als «störend», wenn Fotos oder Videos ohne ihre Zustimmung von anderen Personen online gestellt wurden (ebd. S. 33).

\section{Ausblick}

bildbild ist das Produkt mehrmonatiger Erörterungen, die um diverse Aspekte kreisen:

- die Auswahl der Bildserien im Hinblick auf ihre Anwendbarkeit im Unterricht bzw. ihren Adressatenbezug für Kinder und Jugendliche;

- die schriftliche Adressierung von Schülerinnen und Schülern der Sekundarstufe 1 zur Erläuterung dieses Tools, aber auch zur Anregung, damit zu arbeiten;

- die Adressierung an Lehrpersonen mit Sachinformationen zu den jeweiligen Bildkontexten und den Fotografen/-innen (Abb. 7, 8),

- Methoden der Visual Literacy und, nicht zuletzt,

- um die Gestalt- und Machbarkeit vielfältiger auch interaktiver Wünsche auf der Webseite. 


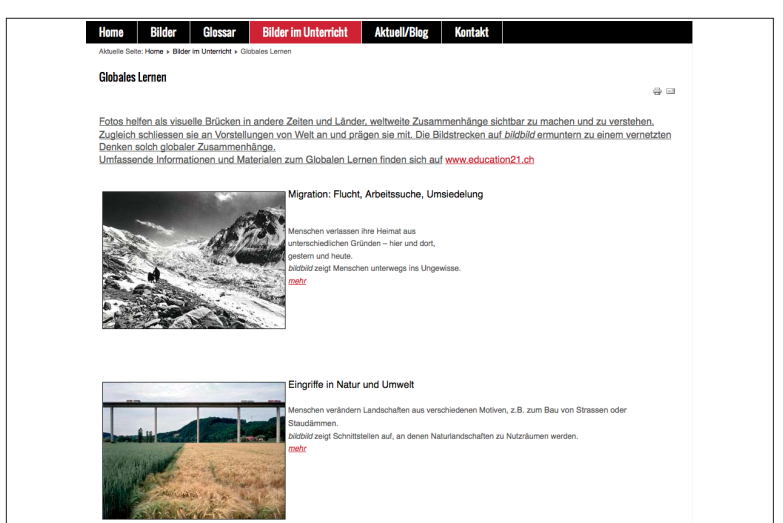

Abb. 7: Informationen zu Globalem Lernen für Lehrpersonen

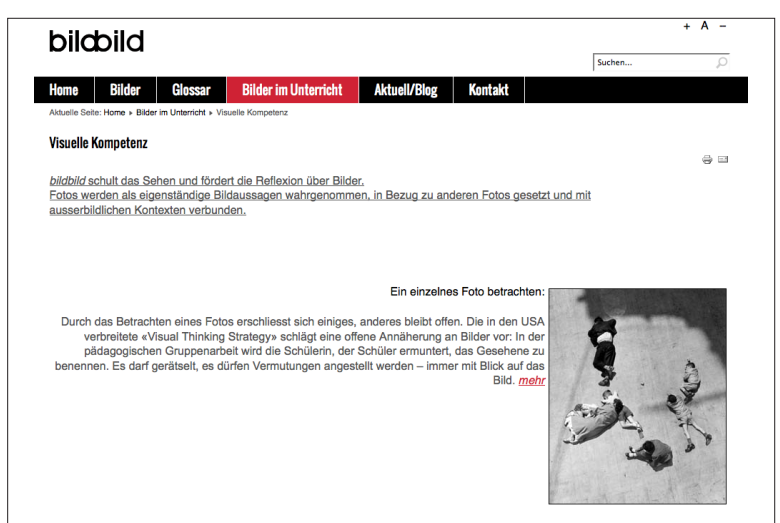

Abb. 8: Informationen zu Visueller Kompetenz für Lehrpersonen

bildbild ist ein Anfang, der weiterer Ergänzungen bedarf. Nicht zuletzt wegen der interdisziplinären Zugänge zu Bildern sind verschiedene Perspektiven noch aufzunehmen oder zu modifizieren. Dazu bedarf es weiterer Resonanz aus der schulischen Praxis. Gerade das Format einer Webseite bietet hierbei Aktualisierungsmöglichkeiten sowie mit einem verlinkten Blog auch interaktive Optionen, die in gedruckten Lehrmitteln so nicht gegeben sind. Lehrpersonen haben dadurch die Möglichkeit, die vielfältigen Angebote von bildbild selektiv und jeweils für ihren Unterricht passend zu nutzen. Sie können den Fokus auf formale Elemente der Bildgestaltung legen und sie vergleichend in anderen Bildserien aufsuchen, etwa Unschärfe, Kontraste, Licht, verschiedene Kameraperspektiven, Fluchtpunkte und Linien oder Bildaufteilungen. Sie können aber auch thematisch verschiedene Bildserien auswählen, um sie im Fachunterricht zu behandeln. Für eine fächerübergreifende Medienbildung bietet bildbild viele Möglichkeiten.

Bilder sind mehr als nur Informationsträger für Erfahrungen zweiter Hand - mitunter sind sie die Quelle schlechthin, um sich Eindrücke fremder Vorgänge zu verschaffen. Sie machen zugleich selbst bei sperrigen politischen oder historischen Themen Bezüge zur unmittelbaren Erfahrung der Schülerinnen und Schüler möglich. Klassische ikonographische Motive können ganz schnell auch mit persönlichen Erlebnissen, mit Wissenserfahrungen der Bildbetrachtenden rückgekoppelt werden, die sie in der Familie, auf Reisen oder anderswo gesammelt haben. Bilder bringen die Welt näher, sind zugleich vieldeutig und offenbaren gerade in der Serie vielfältige oder wiederkehrende Formenstrukturen. Im entschleunigten Betrachten können Schülerinnen und Schüler auf bildbild unterschiedliche Interpretationen der Wirklichkeit, unterschiedliche Denkbilder zu einander in Beziehung setzen sowie Beobachtungen, Benennungen und Deutungen kritisch diskutieren. Das wäre ein Schritt hin zur visuellen Kompetenz zwischen Schwarzweiss-Fotografie, Medienbild und vermeintlich digitaler Selbstverständlichkeit. 


\section{Literatur}

Adorno, Theodor. 1970. Erziehung zur Mündigkeit. Frankfurt/Main: Suhrkamp.

Arnold, Klaus. 2010. "Wie Deutschland begann, sich für Politik zu interessieren: Medienrezeption in den 1960er und 1970er Jahren.» In Von der Politisierung der Medien zur Medialisierung des Politischen? Zum Verhältnis von Medien, Öffentlichkeiten und Politik im 20. Jahrhundert, hrsg. v. Klaus Arnold, Christoph Classen, Susanne Kinnebrock, Edgar Lersch u. Hans-Ulrich Wagner, 323-346. Leipzig: Leipziger Universitätsverlag.

Baudrillard, Jean. 1981. Simulacres et simulation. Paris: Galilée.

Benjamin, Walter. (1936) 2003. Das Kunstwerk im Zeitalter seiner technischen Reproduzierbarkeit. Frankfurt/Main: Suhrkamp.

Boehm, Gottfried. 2007. Wie Bilder Sinn erzeugen: Die Macht des Zeigens. Berlin: University Press.

Christolova, Lena. 2011. «Zwischen den Chiffren von Regnault und der Taxidermie von Flaherty: Wissenschaftsanspruch und Massenkulturphänomene im ethnographischen Film zwischen 1895 und 1931.» In Visuelle Medien und Forschung: Über den wissenschaftlich-methodischen Umgang mit Fotografie und Film, hrsg. v. Irene Ziehe u. Ulrich Hägele. 49-68. Münster: Waxmann.

Debes, John 1969. "The Loom of Visual Literacy.» Audiovisual Instruction 14(8): 25-27.

Doelker, Christian. (1997) 2002. Ein Bild ist mehr als ein Bild: Visuelle Kompetenz in der Multimedia-Gesellschaft. Stuttgart: Klett-Cotta.

Doelker, Christian. 2011. «Visuelle Kompetenz - Grundzüge der Bildsemantik.»In Visuelle Kompetenz: Beiträge des interfakultären Forums, hrsg. v. Theo Hug u. Andreas Kriwak, 9-27. Innsbruck: Innsbruck University Press.

Gehlen, Arnold. (1952) 2004. «Mensch trotz Masse: Der Einzelne in der Umwälzung der Gesellschaft.» In Die Seele im technischen Zeitalter und andere sozialpsychologische, soziologische und kulturanalytische Schriften. Arnold Gehlen-Gesamtausgabe, Bd. 6, hrsg. v. Karl Siegbert Rehberg, 217-228. Frankfurt/Main: Vittorio Klostermann.

Gehlen, Arnold. (1955) 2004. «Massenpsychologie und Sozialpsychologie.». In Die Seele im technischen Zeitalter und andere sozialpsychologische, soziologische und kulturanalytische Schriften. Arnold Gehlen-Gesamtausgabe, Bd. 6, hrsg. v. Karl Siegbert Rehberg, 229-249. Frankfurt/Main: Vittorio Klostermann.

Genazino, Wilhelm. 2004. Der gedehnte Blick. München: Hanser.

Grosvenor, lan, Martin Lawn und Kate Rousmaniere, ed. 1999. Silences and Images: The Social History of the Classroom. New York: Peter Lang.

Grube, Norbert. 2012. «A «New Republic`? The Debate between John Dewey and Walter Lippmann and Its Reception in Pre- and Postwar Germany.» In The Global Reception of John Dewey's Thought: Multiple Refractions Through Time 
and Space (Routledge International Studies in the Philosophy of Education, 28), ed. Rosa Bruno-Jofré and Jürgen Schriewer, 196-214. New York u. London: Routledge.

Habermas, Jürgen. 1990. Strukturwandel der Öffentlichkeit: Untersuchungen zu einer Kategorie der bürgerlichen Gesellschaft. Frankfurt am Main: Suhrkamp.

Heuer, Christian. 2006. «Der 'gedehnte Blick» und die Geschichtsdidaktik.»In Foto + Text: Handbuch für die Bildungsarbeit, hrsg. v. Alfred Holzbrecher, Ingelore Oomen-Welke u. Jan Schmolling, 203-214. Wiesbaden: Verlag für Sozialwissenschaften.

Kracauer, Siegfried. (1927) 1990. «Die Photographie.»In Schriften, Bd. 5.2. Aufsätze (1927-1931), hrsg. v. Inka Mülder-Bach, 83-98. Frankfurt/Main: Suhrkamp.

Machill, Marcel und Felicitas von Peter, Hrsg. 2001. Internet-Verantwortung an Schulen. Gütersloh: Verlag Bertelsmann Stiftung.

Merzyn, Gottfried. 1994. Physikschulbücher, Physiklehrer und Physikunterricht: Beiträge auf der Grundlage einer Befragung westdeutscher Physiklehrer. Kiel: Institut für die Pädagogik der Naturwissenschaften.

Moholy-Nagy, Laszlo (1936) 1981. «From Pigment to Light.» In Photography in print: Writings from 1816 to the present, ed. Vicki Goldberg, 339-348. New York: Simon and Schuster.

Nolte, Paul. 2000. Die Ordnung der deutschen Gesellschaft: Selbstentwurf und Selbstbeschreibung im 20. Jahrhundert. München: Beck.

Panofsky, Erwin. 1975. «lkonographie und Ikonologie: Eine Einführung in die Kunst der Renaissance.» In Sinn und Deutung in der bildenden Kunst, 36-67. Köln: Dumont.

Pfrunder, Peter und Martin Gasser, Hrsg. 2003. Fokus 50er Jahre: Yvan Dalain, Rob Gnant und ¿Die Woches. Zürich: Limmat Verlag.

Pilarczyk, Ulrike und Ulrike Mietzner. 2005. Das reflektierte Bild: Die seriell-ikonografische Fotoanalyse in den Erziehungs- und Sozialwissenschaften. Bad Heilbronn: Julius Klinkhardt.

Radkau, Joachim. 1998. Das Zeitalter der Nervosität: Deutschland zwischen Bismarck und Hitler. München: Carl Hanser Verlag.

Sarasin, Philipp. 2011. «Was ist Wissensgeschichte?» Internationales Archiv für Sozialgeschichte der deutschen Literatur 36 (1): 159-172.

Schäffer, Burkhard. 2005. «Erziehungswissenschaft.»In Bildwissenschaft: Disziplinen, Themen, Methoden, hrsg. v. Klaus Sachs-Hombach, 213-225. Frankfurt/ Main: Suhrkamp.

Schnakenberg, Ulrich. 2012. Die Karikatur im Geschichtsunterricht. Schwalbach: Wochenschau.

Seibert, Andreas. 2008. From Somewhere to Nowhere: China's Internal Migrants. Baden: Müller. 
Sims, Ellen, Ros O'Leary, Jules Cook und Gill Butland. 2002. «Visual Literacy: What is it and do we need it to use learing technologies effectively?» http://www. ascilite.org.au/conferences/auckland02/proceedings/papers/ellen_sims_et_ al.pdf [Zugriff 20.3.2013].

Soeffner, Hans-Georg und Jürgen Raab. 2004. «Bildverstehen als Kulturverstehen in medialisierten Gesellschaften.»In Positionen der Kulturanthroplogie, hrsg. v. Aleida Assmann, Ulrich Gaier u. Gisela Trommsdorff, 249-274. Frankfurt/Main: Suhrkamp.

Sontag, Susan. 2011. «Die Bilderwelt.» In Über Fotografie, 146-172. Frankfurt/ Main: Fischer.

Staub, Hans. 1984. Schweizer Alltag: Eine Photochronik 1930-1945 [zur Ausstellung «Hans Staub» im Kunsthaus Zürich, 18. August - 7. Oktober 1984]. Bern: Benteli.

Stumberger, Rudolf. 2010. Klassen-Bilder: Sozialdokumentarische Fotografie. Bd. 2: 1945-2000. Konstanz: UVK.

Tucholsky, Kurt. (1912) 1975. «Mehr Fotografien!» In Gesammelte Werke in zehn Bänden, Bd. 1, hrsg. v. Mary Gerold-Tucholsky u. Fritz J. Raddatz, 47. Reinbek bei Hamburg: Rowohlt.

Willemse, Isabel, Gergor Waller, Daniel Süss, Sarah Genner und Anna-Lena Huber. 2013. JAMES: Jugend, Aktivitäten, Medien - Erhebung Schweiz: Ergebnisbericht zur JAMES-Studie 2012. Zürich: Zürcher Hochschule für Angewandte Psychologie, ZHAW. http://www.psychologie.zhaw.ch/fileadmin/user_upload/ psychologie/Downloads/Forschung/JAMES/JAMES_2013/Ergebnisbericht_ JAMES_2012.pdf [Zugriff 20.3.2013].

\section{Webseiten}

International Visual Literacy Association (IVLA). http://www.ivla.org (u. a. Definition des Begriffs basierend auf John Debes, 1969).

Picture This: California Perspectives on American History. http://collections.museumca.org/?q=category/2011-schema/history/picture (Enthält eine Vielzahl von «Visual Literacy Activities»). 